\title{
Effects of One-day e-learning Education on Perinatal Psychological Support Among Midwives: a Randomised Controlled Study
}

Eriko Shinohara ( $\nabla$ eshino@yokohama-cu.ac.jp )

Yokohama City University

\section{Yukiko Ohashi}

Josai International University

Ayako Hada

Kitamura Institute of Mental Health Tokyo

Yuriko Usui

University of Tokyo

\section{Research Article}

Keywords: Empathy, Communication, Education, Randomised controlled trial, Midwifery

Posted Date: October 28th, 2021

DOI: https://doi.org/10.21203/rs.3.rs-953233/v1

License: (c) (i) This work is licensed under a Creative Commons Attribution 4.0 International License. Read Full License 


\section{Abstract}

Background: Although midwives are expected to play a key role for psychological support throughout perinatal periods, their educational chances are limited. Versatile teaching strategies such as e-learning may be promising in expanding education. The objective of our study was to clarify the effects of an elearning educational programme on midwives' empathic communication skills.

Methods: From April 2019 to September 2019, a randomised controlled trial of a one-day e-learning educational programme on perinatal psychological issues (both perinatal mental health assessment and empathic communication) was conducted to improve empathic communication skills of midwives. Two types of measurements (paper-and-pencil multiple-choice test and video-viewing tests of simulated patient) were used to measure the competency of empathic communication skills.

Results: Participants $(N=115)$ were randomly allocated to two groups (Intervention: $n=58$, Control: $n=$ 57). The intervention group was at a significantly higher level for both post-tests of empathic communication skills compared with the control group. Both intervention and control groups showed improvements in acquiring knowledge about perinatal mental health assessments.

Conclusions: The results of our study show that a one-day e-learning programme helped improve the midwives' empathic communications skills. Therefore, an effective one-day e-learning educational programme of perinatal mental health will expand opportunity to learn about empathic communication skills for midwives.

Trial registrations: UMIN000036052

\section{Background}

The perinatal period is characterised by the onset of various mental health problems due to role changes in women's lives [1, 2] or dramatical hormonal change [3]. Among perinatal health professionals, midwives are situated in an important position to provide psychological assessment and support for perinatal women. Midwives are required to have two distinctive types of competencies: assessment and support. Assessment includes knowledge of diagnosis, symptomatology, and epidemiology of mental disorders commonly seen in the perinatal period such as mood, anxiety, psychotic disorders, bonding disorders, and child abuse. As for support, psychological counselling has been shown to be effective in treating postpartum depression [4]. Effective support is based on the midwives' skills of empathic communication [5]. Empathic communication is a basis of psychological intervention [6]. A recent review by Coates and Foureur [7] noted that 'midwives play an important role in the provision of mental health interventions such as counselling'. Nevertheless, despite the importance of such knowledge and skills, little has been studied about an effective and efficient educational system for practicing midwives $[5,8]$. In spite of the need, there are various challenges regarding the issue of perinatal mental health support provided by midwives, such as lack of educational resources or training, and time constrains [9]. To solve these problems, web-based e-learning may be a promising candidate as an educational tool. Access to 
educational content via the internet is simple and does not require travelling to a lecture theatre [10]. In the area of mental health, e-learning is used to train many therapists [11], moreover, e-learning educational interventions have been reported to improve therapist competence and to be cost effective [12].

The use of videos of interview scenes is considered effective as an educational tool to enhance psychological support skills. The use of video materials in psychiatric lecture increases satisfaction and long-term retention for students [13]. It can demonstrate near-real 'pictures' as to how to assess mental states and how to have an intervention interview. As a means of judging the effects of an educational system, paper-and-pencil tests may be suitable for assessing knowledge but not suitable for assessing clinical skills such as empathic communication. The perceived competence or satisfaction with the courses of students and clinicians who receive training may not be appropriate as a measure of their clinical skills. For example, Neumann [14] studied the relationship between students' satisfaction with their courses and their performance and found a positive correlation between the two among engineering students but no correlation among medical students. Rodin and Rodin [15] studied undergraduate students whose amount learned from an instructor and the students' evaluation of the instructor's teaching performance was negatively correlated. These studies cast doubt whether asking about students' perceived competency would reasonably reflect their actual performance. Hence, role play tests may be more appropriate to evaluate the skills. Here, a student or a clinician has a mock interview with an examiner who takes the role of a patient or client. However, this is very time-consuming and difficult to standardise (i.e. the examiner's reaction may differ from one test candidate to another). An alternative method may be showing a short-recorded interview session (video-viewing test of simulated patient interviewed by a midwife) and asking to note what the candidate would say to the patient after watching the recording. This video-viewing test is akin to a mock interview examination but more standardised and easier to quantify the candidate's level of empathic communication.

The main purpose of this study was to evaluate the effects of e-learning education for empathic communication skills rated using a video-viewing test. The secondary purpose was to assess the effects of e-leaning education for empathic communication skills rated using paper-and-pencil multiple-choice tests, perinatal mental health knowledge, and attitudes towards support for women in perinatal periods.

\section{Methods}

\section{Study design and procedure}

This was an open-label, parallel-arm randomised controlled trial. We invited midwives, nurses, and public health nurses who had roles in perinatal care to participate in a one-day e-learning course of perinatal mental health education. The participants were randomly divided into two groups: Groups A (intervention) and $B$ (control). The contents of the e-learning consisted of two parts: perinatal mental health assessment and empathic communication. They viewed the same educational contents, but the timing of measurement was different. The perinatal mental health assessment part was learned in the morning 
(about two hours) and the empathic communication part in the afternoon (about two hours). The same paper-and-pencil multiple-choice test (empathic communication skills and assessment knowledge), video-viewing test (empathic communication skills), and an attitude test were conducted before the morning session (the pre-tests) in both Groups A and B. Similar tests were performed in the afternoon (the post-tests). The post-tests were performed before viewing the empathic communication part for Group B participants and after the viewing for Group A participants.

We compared the participants' level of empathic communication skills between Group A and Group B. The sessions took place in Oita (April 2019), Yokohama (April 2019), and Sendai (June 2019). Participants who were included in the study submitted their signed consent form to the researcher.

\section{Participants}

Participants were midwives, nurses, and public health nurses who had roles in perinatal care. In Japan, in addition to midwives, obstetric nurses, and public health nurses also provide psychological support to women during the perinatal period. Therefore, such professionals other than midwives were included in this study. Participants were excluded if they had taken the same e-learning course before the study or were aged over 80 , since the rate of internet use drops sharply for people over 80 years old, they are considered as difficult target population for e-learning education in Japan [16]. We recruited participants by sending a study recruitment letter and study consent form to midwife associations, hospitals, clinics, and birth centres in Oita Prefecture, Yokohama City, and Sendai City during periods of February 2019 through May 2019.

\section{Randomisation and masking}

Randomisation was performed with a random chart with a block size of four. A consecutive number of sealed opaque envelopes with a randomisation letter (A or B) were prepared by the researcher. Envelopes were opened by research assistants when the participants came in for the seminar, who were then allocated to one of the two groups. Due of the characteristics of intervention, masking was not applicable in this trial.

\section{Educational content}

The present educational course was developed by the T. and F. Kitamura Foundation of Studies and Skill Advancement in Mental Health (Tokyo). The material was recently developed for clinical midwives, as well as other perinatal health professionals, to acquire knowledge and gain skills of empathic communication in perinatal mental health issues. Both the perinatal mental health assessment and empathic communication section s consist of eight 15-minute video scripts. The perinatal mental health assessment section described (a) diagnostic criteria and the importance of mental health assessment, (b) the epidemiology of mental disorders commonly seen in the perinatal period, (c) mood disorders, (d) anxiety disorders, (e) psychotic disorders and puerperal psychosis, (f) child abuse and intimate partner violence, $(\mathrm{g})$ perinatal bonding disorders, and $(\mathrm{h})$ perinatal grief. The empathic communication section 
described (a) preparation for a mental health interview, (b) basic interview skills, (c) active listening, (d) probe techniques, (e) presentation of a case of acute insomnia after childbirth, and (f) catharsis.

\section{Outcomes}

The primary outcome was improvement of skills of empathic communication rated using a video-viewing test of a simulated patient interviewed by a midwife (SEC-V). Secondary outcomes were improvement in the skills of empathic communication rated using paper-and-pencil multiple-choice tests (SEC-P), knowledge of perinatal mental health assessment rated using paper-and-pencil multiple-choice tests (KPMHA), and attitudes towards support for women in perinatal periods.

\section{Measurement}

Demographic information and other variables: The participants were asked their age, years of clinical experience, educational background, and job title. These questions were asked in the pre-test.

Empathic communication skills: We used two types of measurements about the participants' competency to empathically communicate with pregnant women. One was a paper-and-pencil multiple-choice test with five questions to evaluate the skills of empathic communication (SEC-P). Here, a short case vignette was presented with four choices for the best empathic response. One point was given for the correct answer whereas the other answers received zero points. The total score ranged from 0 to 5 .

The second was a video-viewing test of a simulated patient interviewed by a midwife to evaluate the skills of empathic communication (SEC-V). A short (approximately 5 minutes long) video script (for example see Appendix) was shown and was followed by the question 'If you were in charge of the care of this woman, what would you say to her? Give your response verbatim'. The degree of empathy of each narrative was rated objectively by evaluators (ES, AH, and $\mathrm{YU}$ ) using the Empathic Understanding in Interpersonal Processes scale (EUIP [17]). The EUIP has a single item which is rated using a 5-point scale from not empathic at all (1) to very empathic (5). Low scores on the EUIP indicate that the participant's utterance is irrelevant to or even antagonising the patient's emotion while higher scores on the EUIP indicate that their utterance is in accordance with the patient's emotion or even leading the patient into deeper insight. This evaluation was performed in the pre- and post-tests using different video scripts. In order to check interrater reliability of the EUIP assessment, the three raters independently rated the verbatim responses of $20 \%$ of the participants. Interrater agreement was $\mathrm{K}=.80$. In the remaining $80 \%$ of cases' responses were rated by the three raters separately.

Knowledge of perinatal mental health assessment (KPMHA): To assess the knowledge of perinatal mental health, a pencil-and-paper multiple-choice test with 10 questions, each with a choice of four answers, was administered. Questions were created by experts in perinatal mental health. One point was given for the correct answer whereas the other answers received zero points. The score ranged from 0 to 10. To ensure an equivalent level of difficulty for pre-and post-tests, pilot-tests of 82 questions were conducted among six midwives who were not eligible participants to calculate the rate of correct 
answers, and 30 questions ( 15 each for pre-and post-tests) of the same level of difficulty were chosen. The final versions were asked in the pre- and post-tests.

Attitude for psychological support: We used the Counsellor Response Form (CRF [18]) to measure the participant's active attitude towards providing psychological support to the case described in the video script. The CRF has a single item and is rated using a 9-point scale, 'Do not want support her' (1) to 'Want to support her' (9). This was asked in the pre- and post-tests.

\section{Ethical considerations}

The study protocol was approved by the Institutional Review Board of Tokyo Healthcare University (Kyou30-35C) and registered with UMIN-CTR in Japan (UMIN000036052; dated 1, March 2019). This clinical trial adhered to the Code of Ethics of the World Medical Association and the clinical research ethical guidelines for human subjects established on 27 April 2015, by the Ministry of Education, Culture, Sports, Science and Technology, and the Ministry of Health, Labour and Welfare, Japan.

\section{Sample size and power}

There have been few previous studies of this kind. Referencing a study using the EUIP [19], based on 95\% power to detect a significant difference ( $a=0.05$, two-sided), 164 participants were required.

\section{Statistical analysis}

We used two-way analysis of variances (ANOVAs) to examine the main effects of the group (intervention vs control) and the timing (pre- vs post-tests) and their interactive terms on the scores of SEC-V, SEC-P, KPMHA and attitude. All statistical analyses were conducted using SPSS version 26.0.

\section{Results}

A total of 134 women agreed to participate, of whom 115 were randomly allocated to the Group A $(n=$ $58)$ or Group $B(n=57)$. Figure 1 displays a flow diagram of trial recruitment and follow-up. All participants were followed and included to analysis. Participants were midwives $(n=105)$, nurses $(n=5)$, and public health nurses $(n=5)$. The participants' age, duration of clinical practice, and education were in Table 1. 
Table 1

Backgrounds of participants

\begin{tabular}{|llll|}
\hline & $\begin{array}{l}\text { Group A: Intervention } \\
(\mathbf{n}=\mathbf{5 8}) \\
M(S D)\end{array}$ & $\begin{array}{l}\text { Group B: Control } \\
(\mathbf{n}=\mathbf{5 7})\end{array}$ & $P(95 \% \mathrm{Cl})$ \\
& & $\boldsymbol{M ( S D )}$ & \\
\hline Age & $47.3(12.7)$ & $45.8(12.9)$ & $.521(-3.20-6.29)$ \\
\hline Years & $20.1(11.9)$ & $17.3(12.5)$ & $.226(-1.74-7.29)$ \\
Education & & & .495 \\
College & 29 & 28 & \\
Junior-college & 10 & 6 & \\
University & 10 & 16 & \\
Graduate school & 8 & 8 & \\
\hline Abbreviations: $M$, mean; SD, standard deviation & \\
\hline
\end{tabular}

The SEC-V and SEC-P were moderately correlated, $r=.409, p<.001$. For the primary outcome, the scores of the SEC-V (EUIP) showed the main effects of the group $(F(1)=4.302, p<.05)$, timing $(F(1)=21.967, p$ $<.001)$ as well as the interactive effect $(F(1,1)=25.157, p<.001)$. For the secondary outcome, the scores of the SEC-P showed the main effects of the group $(F(1)=32.364, p<.0001)$ and timing $(F(1)=32.364, p$ $<.001)$. Their interactive effect was also significant $(F(1,1)=40.269, p<.001)$. Thus, both the SEC-V and SEC-P scores were higher among the intervention group participants at the post-test occasion (Table 2). These findings indicated that the present programme improved the participants' empathic communications. 
Table 2

Psychological skills between groups

\begin{tabular}{|lllll|}
\hline \multicolumn{4}{|c|}{ Group A: intervention } & \multicolumn{3}{l|}{ Group B: control } \\
& Pre-test & Post-test & Pre-test & Post-test \\
& $M(S D)$ & $M(S D)$ & $M(S D)$ & $M(S D)$ \\
\hline ECS-V(EUIP) & $1.81(0.79)$ & $2.82(0.91)$ & $2.07(0.86)$ & $2.03(0.97)$ \\
\hline ECS-P & $4.20(1.12)$ & $5.48(0.81)$ & $4.49(0.89)$ & $4.42(0.76)$ \\
\hline KPMHA & $4.07(1.24)$ & $8.04(1.12)$ & $3.95(1.34)$ & $8.05(0.97)$ \\
\hline Attitude (CRF) & $7.96(1.14)$ & $8.11(1.03)$ & $8.20(0.98)$ & $8.09(1.01)$ \\
\hline $\begin{array}{l}\text { Abbreviations: M, mean; SD, standard deviation; ECS-V, Empathic Communication Skills (Video- } \\
\text { viewing tests of simulated patient interviewed by a midwife); EUIP, Empathic Understanding in } \\
\text { Interpersonal Process; ECS-P, Empathic Communication Skills (Paper-and-pencil multiple-choice } \\
\text { tests); KPMHA, Knowledge of Perinatal Mental health Assessment (Paper-and-pencil multiple-choice } \\
\text { tests); CRF, Counsellor Response Form. }\end{array}$ & & \\
\hline
\end{tabular}

The other secondary outcome, KPMHA scores showed the main effect of timing $(F(1)=891.081, p<.001)$ but no main effect of the group $(F(1)=0.092$, NS). No interactive terms were significant $(F(1,1)=0.270$, NS). Therefore, the KPMHA of both the intervention and control groups improved after the course. For attitude, no main effect was observed for either group $(F(1)=2.998, N S)$ or timing $(F(1,1)=0.061, N S)$.

\section{Discussion}

\section{Main findings}

Our results indicated that the one-day e-learning educational programme of perinatal mental health issues was effective in improving the midwives' empathic communication skills with pregnant women. In addition, this one-day e-learning programme enhanced the participants' knowledge about perinatal mental health assessment. As expected, both the intervention and control groups showed improvement in knowledge of perinatal mental health problems whereas only the intervention group showed a marked increase in empathic communication skills. This may support the educational effects of the skills part of the programme. This positive result may come from the design of the educational programme in which the participants learn perinatal mental health assessment before the empathic communication skills. Since knowledge is a factor underlying psychological support attitudes [19], combination of both knowledge and skills may have synergistic effects for empathic communication skills. Another strength of this study is the use of many video scripts describing vignettes of different mental health problems (e.g. mood and anxiety disorders, bonding disorders, and child abuse) as well as role plays of empathic communication interviews. These may give a more realistic picture of clinical knowledge and skills than texts in leaflets and books. Video materials incorporated in psychiatric lectures have been reported to 
increase students' satisfaction and long-term retention [13]. Our study also used videos of patients for evaluation of empathic communication skill. Simulated patients are used as a means of evaluation in the objective structured clinical examinations (OSCEs). They are often used in clinical education, and it is thought to be appropriate to assess communication skills [20]. However, OSCEs need the staff and time for examinations. In the present study, e-learning did not require many staff and required only less time for preparation. Therefore, using e-learning for education and evaluation may be more convenient than OSCEs.

Of research and clinical interest are the only modest correlation between participants' competency rated by SEC-P and SEC-V. In the former, the participants were requested to select a response which they thought as the most empathic. This is a reflection of mere 'understanding' of empathic communication. In the latter, however, they were requested to spontaneously 'invent' a verbatim response to an imaginary patient. This is a reflection of their skills of empathic communication. The SEC-V may be more difficult to answer but realistically similar to a clinical setting. Therefore, we think that the SEC-V is a better measure to reflect the participants' clinical skills of empathic communication.

Midwives feel a lack of skills and confidence for perinatal mental health care [7]. There is demand for learning needs for perinatal mental health and care [21], although one barrier is lack of accessible educational resources [9]. There are few educational research reports specific to psychological support for perinatal mental health. A series of psychological support education seminars for midwives and nurses were effective in a previous study [5]. However, two-days training was not effective for midwives [8]. Unlike Saito et al.'s study which was designed as a several-day course, the present study used only one-day e-leaning material. The most effective duration for intervention was over four hours [22]. We used a four-hour e-learning course. Although the duration of our course was shorter than that used in the previous study [5] [8], this course is promising as means of the mental health education for busy perinatal health professionals. The e-learning used in this study substantially improved midwives' and nurses' perinatal mental health assessment knowledge as well as empathic communication skills. The e-learning educational tool used in the present study may be promising in delivering education to many experts regardless of time and place in a very short time. This is a very useful learning method, especially under the recent COVID-19 epidemic.

\section{Strength and Limitation}

This was the first randomized controlled trial of e-learning education that specialized in perinatal psychological support. This study had several strengths. The design of the randomized controlled trial reduced spurious causality and bias. In addition, the evaluation of empathic communication was objective, as it was conducted by three experts and inter-raters to ensure credibility, as empathy is considered to be a subjective variable. However, this study did have some limitations. First, the participants responded to our request and were therefore likely to be highly enthusiastic about learning about perinatal mental health issues and psychological support. This may have contributed to the favourable effects of this intervention. Thus, the effects for midwives or nurses with less interest in perinatal mental health issues may have been lower, compared to the estimates of this report. Second, 
the e-learning material was provided in a one-day seminar. The results may differ if the course materials were accessed through online mediums by the participants, depending on their convenience. In addition, the participants in this study were clinicians. Since perinatal mental health education is an important topic for midwifery and nursing students, this study should be replicated with undergraduate students. Third, the sample size was smaller than expected. Further research with a larger sample size is required. In addition, the long-term effects of the educational programme are unknown, as it was conducted over a short interval.

\section{Conclusion}

The present educational programme improved the participant midwives' empathic communication skills. The one-day e-learning education of empathic communication skills used in this study was effective and efficient in improving midwives' supportive skills. Busy clinical midwives are recommended to improve their communication skills in a short duration via e-learning educational materials that are well designed. E-learning education method may pave the way for efficient and effective education about mental health care.

\section{Abbreviations}

SEC-V - video-viewing test of simulated patient interview by a midwife

SEC-P - paper-and-pencil multiple-choice test

KPMHA - knowledge of perinatal mental health assessment

EUIP - Empathic Understanding in Interpersonal Processes scale

CRF - Counsellor Response Form

ANOVA - Analysis of variance

OSCE - objective structured clinical examination

\section{Declarations}

\section{Ethical approval and consent to participate}

All study participants provided informed consent, and the study design was approved by the Institutional Review Board of Tokyo Healthcare University, Tokyo, Japan (Approval no. Kyou30-35C). The date of approval was 17, Jan 2019.

\section{Consent for publication}

Not applicable. 


\section{Data availability statement}

The datasets used and/or analysed during the current study are available from the corresponding author on reasonable request.

\section{Competing interest}

The authors declare that they have no competing interests.

\section{Funding}

This study was funded by the JSPS KAKENHI (Grant No. 18K17585).

\section{Authors' contributions}

E.S. and Y.O. set up the research design. E.S. and A.H. collected data. E.S., A.H., and Y.U. analysed data. E.S. wrote the manuscript.

\section{Acknowledgements}

The authors would like to thank all of the participants, the Oita Midwives Association, and the Yokohama Midwives Association. We are also grateful for Dr. Shoji Sato, Oita Prefectural Hospital, Professor Kineko Sato, Akamon College of Sendai, and Mrs. Tomoko Ito, Tomoko Birth Centre for their support for this study. Thanks are due to the T. and F. Kitamura Foundation of Studies and Skill Advancement in Mental Health for their generous preparation of the educational materials.

\section{References}

1. Takegata M, Ohashi Y, Haruna M, Kitamura T. Theoretical framework for interpersonal psychotherapy in the prevention of postpartum depression: a commentary. Int J Nurs Health Sci. 2014;1(6):37-40.

2. Takegata M, Haruna M, Kitamura T. Continuous education for perinatal mental health among clinical midwives: necessity and a literature review. Int J Nurs Health Sci. 2015;2(6):73-7.

3. O'Hara MW, Schlechte JA, Lewis DA, Wright EJ. Prospective study of postpartum blues: biologic and psychosocial factors. Arch Gen Psychiatry. 1991;48(9):801-6. doi:

10.1001/archpsyc.1991.01810330025004, Pubmed:1929770.

4. Stuart S, O'Hara MW, Gorman LL. The prevention and psychotherapeutic treatment of postpartum depression. Arch Womens Ment Health. 2003;6(2);Suppl 2:S57-69. doi: 10.1007/s00737-003-0003-y, Pubmed:14615924.

5. Saito T, Takeda S, Yamagishi Y, Kubo R, Kitamura T. Psychotherapy training on psychological mindedness in a Japanese nurse population: effects and personality correlates. Healthcare (Basel). 2017;5(3):43. doi: 10.3390/healthcare5030043, Pubmed:28786921. 
6. Egan G, Reese RJ. The skilled helper: a problem-management and opportunity-development approach to helping. 11th ed. Stanford, Connecticut: Cengage Learning; 2018.

7. Coates $D$, Foureur $M$. The role and competence of midwives in supporting women with mental health concerns during the perinatal period: a scoping review. Health Soc Care Community. 2019;27(4):e389-405. doi: 10.1111/hsc.12740, Pubmed:30900371.

8. Alhassan M. Effect of a 2-day communication skills training on nursing and midwifery students' empathy: a randomised controlled trial. BMJ Open. 2019;9(3):e023666. doi: 10.1136/bmjopen-2018023666, Pubmed:30826757.

9. Bayrampour H, Hapsari AP, Pavlovic J. Barriers to addressing perinatal mental health issues in midwifery settings. Midwifery. 2018;59:47-58. doi: 10.1016/j.midw.2017.12.020, Pubmed:29353691.

10. Sinclair P, Kable A, Levett-Jones T. The effectiveness of internet-based e-learning on clinician behavior and patient outcomes: a systematic review protocol. JBI Database System Rev Implement Rep. 2015;13(1):52-64. doi: 10.11124/jbisrir-2015-1919, Pubmed:26447007.

11. O'Connor M, Morgan KE, Bailey-Straebler S, Fairburn CG, Cooper Z. Increasing the availability of psychological treatments: a multinational study of a scalable method for training therapists. J Med Internet Res. 2018;20(6):e10386. doi: 10.2196/10386, Pubmed:29884606.

12. Fairburn CG, Allen E, Bailey-Straebler S, O'Connor ME, Cooper Z. Scaling up psychological treatments: A countrywide test of the online training of therapists. J Med Internet Res. 2017;19(6):e214. doi: 10.2196/jmir.7864, Pubmed:28623184.

13. Averbuch R, Garvan C. It works! teaching psychiatry with videos enhances long term retention, plenary session. Res Med Educ. 35th Annual Meeting of ADMSEP (Association of Directors of Medical Student Education in Psychiatry), Portsmouth, NH, 19 Jun 2009.

14. Neumann L. Student evaluation of instruction. A comparison of medicine and engineering. Med Educ. 1982;16(3):121-6. doi: 10.1111/j.1365-2923.1982.tb01070.x, Pubmed:7099046.

15. Rodin M, Rodin B. Student evaluations of teachers. Science. 1972;177(4055):1164-6. doi: 10.1126/science.177.4055.1164:177/4055/1164 (pii ). Pubmed:17847197.

16. Ministry of Internal affairs and communications. Internet usage conditions; 2019. https://www.soumu.go.jp/johotsusintokei/whitepaper/ja/r01/html/nd232120.html. Accessed 5 Apr 2021.

17. Carkhuff R. Helping and human relations: a primer for lay and professional helpers. New York: Holt, Rinehart \& Winston; 1969.

18. Kirchberg TM, Neimeyer RA, James RK. Beginning counselors' death concerns and empathic responses to client situations involving death and grief. Death Stud. 1998;22(2):99-120. doi: 10.1080/074811898201623, Pubmed:10182421.

19. Kanda C. Kangosyokusyano syuusannki utubyou kanjyani taisuru shinriennjyotaidowo kiteisuru youin. [Factors determining the attitude of nurses to psychological support for perinatal depression patients] [Master's thesis]. Tokyo: St. Luke's College of Nursing; 2015. 
20. Cömert M, Zill JM, Christalle E, Dirmaier J, Härter M, Scholl I. Assessing communication skills of medical students in objective structured clinical examinations (OSCE)-a systematic review of rating scales. PLOS ONE. 2016;11(3):e0152717. doi: 10.1371/journal.pone.0152717, Pubmed:27031506.

21. Noonan M, Jomeen J, Galvin R, Doody O. Survey of midwives' perinatal mental health knowledge, confidence, attitudes and learning needs. Women Birth. 2018;31(6):e358-66. doi:

10.1016/j.wombi.2018.02.002, Pubmed:29454664.

22. Winter R, Issa E, Roberts N, Norman RI, Howick J. Assessing the effect of empathy-enhancing interventions in health education and training: A systematic review of randomised controlled trials. BMJ Open. 2020;10(9):e036471. doi: 10.1136/bmjopen-2019-036471, Pubmed:32978187.

\section{Figures}

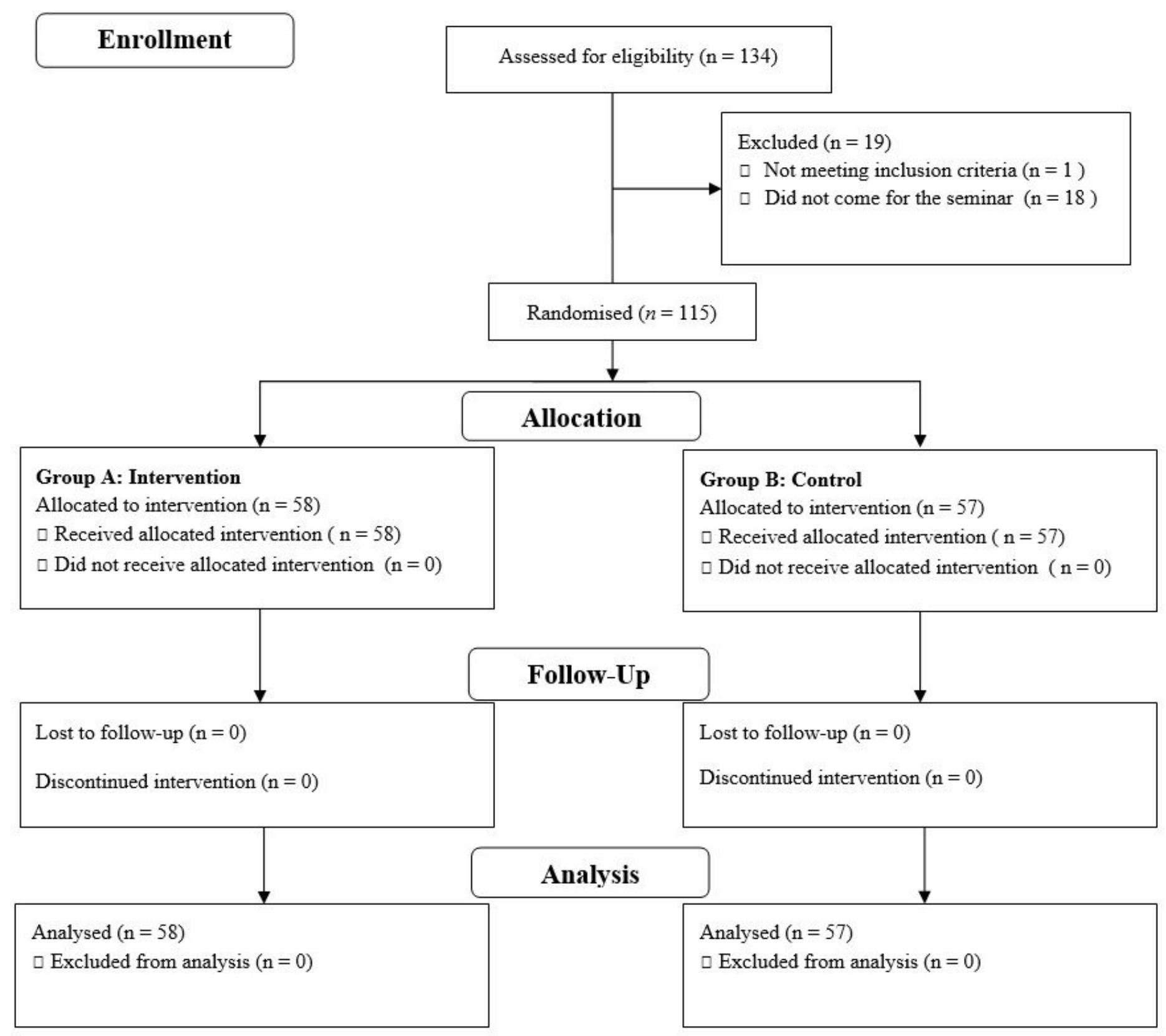




\section{Figure 1}

Flow diagram of the trial.

\section{Supplementary Files}

This is a list of supplementary files associated with this preprint. Click to download.

- BMCadditionalfile1.doc 